\title{
On a new finding of Euciroa arenosa (Rayneval in Appelius, 187I) for the Lower Pliocene of the Mediterranean Basin (Mollusca Bivalvia Euciroidae)
}

\author{
Mauro M. Brunetti' \& Ermanno Quagiotto² \\ ${ }^{1}$ Calle Navas 106, 14511 Las Navas del Selpillar, Spain; e-mail: mbrunetti45@gmail.com \\ ${ }^{2}$ Strada del Tormeno 146, Vicenza, Italy; e-mail: ermanno.quaggiotto@libero.it
}

\begin{abstract}
We report the discovery of a valve of Euciroa arenosa (Rayneval in Appelius, 1871) in the Spanish lower Pliocene, species rarely reported and even less depicted previously in the Italian Pliocene. The family Euriciroidae Dall, 1895 is briefly mentioned. The image of the type species, E. elegantissima Dall, 1881, is shown.
\end{abstract}

KEY WORDS Bivalvia; Euciroidae; Pliocene.

Received 24.09.2021; accepted 09.12.2021; published online 30.12.2021

\section{INTRODUCTION}

A right valve of Euciroa arenosa (Rayneval in Appelius, 1871) has been found (Rayneval in Appelius, 1871) during malacological research carried out in the lower Pliocene of the southern Iberian peninsula (Estepona, Malaga, Spain), Arroyo Vaquero, the urban area El Rodeo (Fig. 1). The specimen was found on the surface of the ground in fields that are occasionally plowed for fire prevention purposes. In this locality no type of stratification is visible. The age of the outcrop was deduced from the associated fauna and from the numerous Pliocene bibliography of the area (see for example: Lozano-Francisco et al, 1993, Vera-Peláezet al., 1995; Guerra-Merchán et al., 1996; Lozano-Francisco \& Vera-Peláez, 2002, 2006; Landau et al., 2003, 2004, 2006, 2007, 2009; Aguirre et al., 2005; Landau \& Mulder, 2020).

The genus Euciroa Dall, 1881, which currently counts 13 species (Worms http://www.marine species. org/aphia.php? $\mathrm{p}=$ taxdetails\&id=406060), is chara- cterized by thin, nacreous shells with ra- dial sculpture composed of very small papillae, and large cardinal tooth in the right valve. The animals belonging to this genus are carnivorous, living at great depths, most of them in the Pacific Ocean. For the anatomical characteristics of this genus, see Dall (1895). The only Euciroa currently present in the Atlantic area is the type species of this genus: E. elegantissima Dall, 1881 of which a syntype is shown in Fig. 2. Said specimen is at the Department of Invertebrate Zoology Collections Smithsonian National Museum of Natural History (Washington, D.C., USA). The specimen was dredged off the island of Cuba from a depth of 534 meters.

ABBREVIATIONS. $\mathrm{L}=$ maximum width of the valve; USNM = Department of Invertebrate Zoology Collections Smithsonian's National Museum of Natural History (Washington, D.C, USA); CMB = Collezione Mauro Brunetti.

\section{RESULTS}

Systematics 
Classis BIVALVIA

Subclassis HETERODONTA

Ordo ANOMALODESMATA Dall, 1889

Superfamilia VERTICORDIOIDEA Stoliczka, 1870

Familia EUCIROIDAE Dall, 1895

Genus Euciroa Dall, 1881

type species: Euciroa elegantissima Dall, 1881

Euciroa arenosa (Rayneval in Appelius, 1871) (Fig. 1)

1871. Pecchiolia arenosa Rayneval, Appelius, p. 276, Tav. 6, fig. 4, 4 a-b

1876. Verticordia arenosa (Rayneval) F.L. Appelius, Seguenza G., p. 110

1882. V. arenosa Raineval, Bombicci, p. 60

1893. Verticordia arenosa (Rayn. d. Heck. Ponzi). Foresti, pp. 196-197.

?1893- Verticordia Bertii Foresti. Foresti, p. 197198

2015. Euciroa arenosa (Rayneval in Appelius, 1871) Bertaccini, Gardella \& Tabanelli, p. 3, tav. 1, figg. 3-7.

Descriptions of Euciroa arenosa made subsequently by three authors are here reported.

Seguenza 1876 (p. 110): “Questa grande specie parmi che debba essere di forma pressoché orbicolata, abbastanza convessa, cogli apici convessi, poco sporgenti, ed incurvati verso la lunula, che è cordato-ovaia, ben circoscritta, abbastanza profonda e concava. La superficie è ornata da numerose costole radianti,strette, disuguali, più sottili e più numerose sulla regione anale, appena sporgenti, mache divengono ben distinte perchè ciascuna ornata da papille prominenti, coniche, disposte in serie, e tanto minori quanto più piccola è la costola. Gl'interstizii sono ricoperti da esili granuli della forma stessa. Il margine interno delle valve è finamente striato o increspato, il dente è grosso, conico, sporgente, incurvato, e s'innalza dirigendosi verso l'apice della valva, dal quale lato presenta uno spigolo acuto".

Foresti 1893 (p. 196): "Le valve di questa specie sono molto grosse, presentano nel loro margine ventrale una insenatura verso la regione posteriore, regione che ordinariamente si mostra un poco depressa al contrario della regione anteriore che è sempre un poco più gonfia. Internamente sono vestite di un grosso stato madreperlaceo che lascia, non però internamente, distinguere le impronte muscolari, le quali si mostrano bene impresse; più larga l'anteriore un poco più allungata la posteriore".

Bertaccini et al. 2015, p. 3: "Conchiglia fragile, madreperlacea, equivalve, inequilaterale, dalla concavitàmolto accentuata. La sua forma varia da circolare ad ovoidale, conseguentemente il valore del rapporto altezza/larghezza oscilla fra 1,13 e 0,89. Il margine anteriore è leggermente incavato. La superficie esterna è percorsa da numerosissime linee di accrescimento e da linee radiali microscopiche non sempre evidenti, inoltre, è contraddistinta dalla presenza di molteplici piccole protuberanze più fitte versoil bordo. All'interno il margine appare liscio. La valva destra presenta nell 'area cardinale, sotto l'umbone, un vistoso dente, mentre la valva sinistra mostra i margini anteriore e posteriore, deviare verso l'interno in prossimità dell'area cardinale, assumendo l'aspetto di due denti allungati e disgiunti dalla piastra. Sotto l'umbone si nota una lamina triangolare con al centro una piccola protuberanza".

\section{DISCUSSION}

It is the first time that this interesting species has been reported for the Pliocene in this area of the Mediterranean Basin.

Please refer to Bertaccini et al. (2015) regarding the interesting information on the troubled history of this species. Our specimen is, in its form, almost identical to the one illustrated by Bertaccini et al. (2015: table 1 fig. 4). The only difference is the nacreous inner layer which is thinner. However, this could be due to different types of fossilization. In fact, the Spanish specimen was found in predominantly sandy soils unlike all the other specimens, found in deep clays.

Among the current species, E. arenosa (Rayneval in Appelius, 1871) has some similarities with E. pacifica Dall, 1895 of the Hawaiian Islands. A syntype of the latter, deposited at the USNM, is depicted (Fig. 3). It differs from $E$. arenosa especially for the more prominent radial sculpture and the cardinal tooth of the smaller right valve. Based on its description, Verticordia bertiii Foresti, 1893, which the author does not depicted, could perhaps represent a youthful form of $E$. arenosa. This can only be ascertained by examining the type material. 


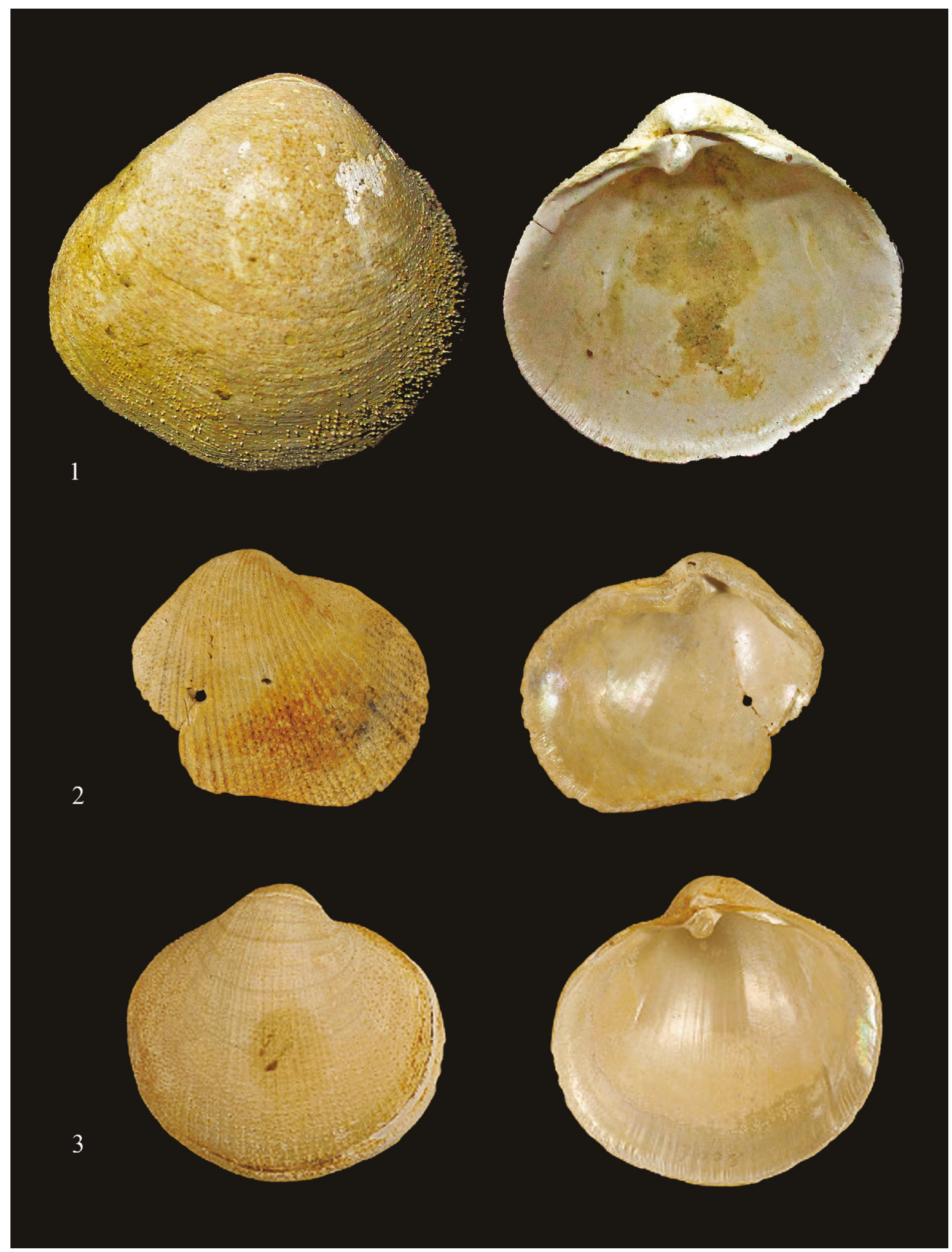

Figure 1. Euciroa arenosa (Rayneval in Appelius, 1871), Lower Pliocene, Arroyo Vaquero (Estepona, Malaga, Spain), L $=32$ mm, CMB. Figure 2. Euciroa elegantissima Dall, 1881, Syntype, Recent, Cuba, -534, L = 23 mm, USNM63217. Figure 3. Euciroa pacifica Dall, 1895, Syntype, Recent, Island Oahu, Hawaii, -539, L = 28 mm, USNM107008. 


\section{REFERENCES}

Appelius F.L., 1871. Catalogo delle conchiglie fossili del Livornese desunto dalle collezioni e manoscritti del defunto G.B. Caterini. Bullettino Malacologico Italiano, 3: 169-297.

Aguirre J., Cãchao M., Domènech R., Lozano-Francisco M. C., Martinell J., Mayoral E., Santos A., VeraPeláez J .L. \& da Silva M.C., 2005. Integrathed Biocronology of the Pliocene deposits of the Estepona Basin (Malaga, S-Spain). Palaeobiogeografic and Palaeoceanographic implications. Revista Española de Paleontología, 20: 225-244.

Bertaccini E., Gardella F. \& Tabanelli C., 2015. Due specie poco conosciute dal Pliocene romagnolo (Mollusca: GatropodaTrochidae, Bivalvia Euciroidae). Quaderno di Studi e Notizie di Storia Naturale della Romagna, 42: 1-8.

Bombicci L., 1882. Montagne e vallate del territorio di Bologna, Cenni sulla oro-idrografia, geologia litologia e mineralogia dell'appennino Bolognese e sue dipendenze. Bologna, Fava \& Garavagni, 208 pp.

Dall W.H., 1895. Report on the Mollusca and Brachiopoda dredged in deep water, chiefly near the Hawaiian Islands, with illustrations of hitherto unfigured species from Northwest America. Proceedings of the United States National Museum, 17: 675-733.

Foresti L., 1893. Enumerazione dei brachiopodi e dei molluschi pliocenici dei dintorni di Bologna. Bullettino della Società Malacologica Italiana, 18: 55-72, $185-413$

Guerra-Merchán A., Palmaqvist P.., Lozano Francisco M.C., Vera Peláez J.L. \& Triviño Rodríguez A., 1996. Análisis sedimentológico y paleoecológico del yacimiento plioceno de Parque Antena (Estepona, Málaga). Revista Española de Paleontología, 11: 226-234.

Landau B., Marquet R. \& Grigis M., 2003. The early Pliocene Gastropoda (Mollusca) of Estepona southern Spain, part 1 Vetigastropoda. Paleobentos, 3: 187.

Landau B., Marquet R. \& Grigis M., 2004. The Early Pliocene Gastropoda (Mollusca) of Estepona Southern Spain. Part 2: Orthogastropoda, Neotaenioglossa. Palaeontos, 4: 1-108.

Landau B. \& Fehse D., 2004. The Early Pliocene Gastropoda (Mollusca) of Estepona Southern Spain. Part 3: Trivoidea, Cypraeoidea. Palaeontos, 5: 1-34.

Landau B., Beu A. \& Marquet R., 2004. The Early Pliocene Gastropoda (Mollusca) of Estepona Southern Spain. Part 4: Tonnoidea, Ficoidae. Palaeontos, 5: 35-102.
Landau B. \& Silva C.M. da., 2006. The early Pliocene Gastropoda (Mollusca) of Estepona southern Spain, part 9: Olividae. Paleobentos, 9: 61-101.

Landau B., La Perna R. \& Marquet R., 2006. The early Pliocene Gastropoda (Mollusca) of Estepona southern Spain, part 11: Marginellidae, Cystiscidae. Paleobentos, 9: 22-60.

Landau B., Petit R. \& Marquet R., 2006. The early Pliocene Gastropoda (Mollusca) of Estepona southern Spain, part 12: Cancellarioidea. Paleobentos, 9: 61-101.

Landau B., La Perna R. \& Marquet R., 2006. The early Pliocene Gastropoda (Mollusca) of Estepona southern Spain, part 6: Triphoroidea, Epitonioidea, Eulimoidea. Palaeontos, 10: 1-96.

Landau B., Houart R. \& Da Silva M., 2007. The early Pliocene Gastropoda (Mollusca) of Estepona southern Spain, part 7: Muricidae. Palaeontos, 11: 1-87.

Landau B., Silva C.M. da \& Gili C., 2009. The early Pliocene Gastropoda (Mollusca) of Estepona southern Spain, part 8: Nassariidae. Palaeontos, 17: 1-101.

Landau B., Da Silva C.M. \& Mayoral E., 2011.The Lower Pliocene gastropods of the Huelva Sands Formation, Guadalquivir Basin, Southwestern Spain. Palaeofocus, 4: 1-90.

Landau B. \& Mulder H., 2020. Additions and corrections to the Gastropod fauna of the Pliocene of Estepona, southwestern Spain. Basteria, 84: 26-57.

Lozano-Francisco M.C., Vera-Peláez J.L. \& GuerraMarchán A., 1993. Arcoida (Mollusca, Bivalvia) del Plioceno de la provincia de Málaga, España. Treballas de Museu Geologico de Barcelona, 3: 157-188.

Lozano-Francisco M.C. \& Vera-Peláez J.L., 2002. Estudio preliminar del Orden Archeogastropoda (Gastropoda, Prosobranchia) del Plioceno della cuenca de Estepona (Málaga, S. España) con la descripción de doce especies nuevas. Pliocenica, 2: 157-175.

Lozano-Francisco M.C. \& Vera-Peláez J.L., 2006. Catálogo del material tipo del Material tipo del Museo Municipal Paleontológico de Estepona, Parte I. Mollusca, Gastropoda. Descripción de nuevas especies del Plioceno de las Cuenca de Estepona (Malaga) y del Guadalquivir (Huelva). Pliocenica, 5: 105-124.

Seguenza G., 1876. Cenni inlorno alle Verticordie fossili del Pliocene Italiano. Rendiconto della Reale Accademia delle Scienze fisiche e matematiche, 15: 104-112.

Vera-Peláez J.L., Lozano-Francisco M.C., Muñiz-Solis R., Gili C., Martinell J., Domènech R., Domènech R., Palmqvist P. \& Guerra-Merchán A., 1995. Estudio preliminar de la malacofauna del Plioceno de Estepona (Málaga, España). Iberus, 13: 93-117.

WoRMShttp://www.marinespecies.org/index.php $8 / 11 / 2020$ 